\title{
Electron Tomography of PEM Fuel Cell Catalyst Coarsening on Alternate Carbon Supports
}

\author{
Brian T. Sneed, ${ }^{1}$ David A. Cullen, ${ }^{2}$ Kimberly S. Reeves, ${ }^{1}$ and Karren L. More ${ }^{1}$ \\ 1. Center for Nanophase Materials Sciences, Oak Ridge National Laboratory, Oak Ridge, TN, USA \\ 2. Materials Science and Technology Division, Oak Ridge National Laboratory, Oak Ridge, TN, USA
}

As hydrogen fuel cell electric vehicles (FCVs) hit the market, much debate remains over their potential commercial impact in comparison to rechargeable battery-based electric vehicle technologies; however, the legitimate concerns of cost, stability, and performance are being addressed by research and development efforts focused on identifying more efficient and durable materials for the critical oxygen reduction reaction taking place at the cathode of the membrane electrode assembly (MEA). In proton exchange membrane fuel cells (PEMFCs), a typical catalyst consists of Pt or Pt-based alloy nanoparticles supported on conductive nanocarbons embedded with a continuous proton-conducting ionomer film. Recent efforts to improve catalytic activity include the development of highly uniform shape-controlled Pt-Ni and Pt-Co alloy electrocatalysts with highly tailored compositions and structures synthesized through wet chemistry methods [1-4]. To improve fuel cell durability, highly graphitized carbon supports have been developed, which show improved corrosion resistance [5-7]. Despite this progress, these materials remain impractical for industrial scale-up. This is in part due to a lack of understanding of the catalyst and catalyst/support three-dimensional interfacial structure and subsequent materials degradation under the harsh operating conditions within the cathode catalyst layer in PEMFCs. Electron tomography in a scanning transmission electron microscope (STEM) can provide necessary insight regarding the 3D structure of interfaces in state-of-the-art PEMFCs.

An electron tomography study of Pt nanoparticle catalysts supported on three different carbon black (CBs) materials and three different catalyst loadings was conducted to evaluate the catalyst/support interactions. Accelerated stress tests (ASTs) were performed for cathode catalyst layers in MEAs comprised of these catalysts to evaluate Pt catalyst degradation on the different nanocarbon supports. We discuss how high surface area carbon (HSAC) supports (turbostratic) with an inherently high porosity show increased initial Pt dispersion, while low surface area carbon (LSAC) carbons (graphitized) reduce Pt dispersion through increased initial Pt particle contact (agglomeration) at the interfaces between nanocarbon particles, likely due to preferential deposition at graphite edge and defect sites rather than on the flat, hydrophobic graphite basal planes (Figure 1). This information can be used to explain differences in the electrochemical active surface area (ECSA) loss of these catalysts during catalyst cycling ASTs $(0.6-0.95 \mathrm{~V}$ for 30,000 cycles), where coalescence is the major contributor to coarsening on the low surface area carbon support, and conversely, Pt dissolution/reprecipitation appears to be the major factor governing ECSA loss of Pt on high surface area carbons. Furthermore, in comparing the 2D-derived Pt crystallite size to the 3D-derived Pt nanoparticle metrics, the latter is found to be a better predictor for ECSA loss, which is attributed to better quantification of initial Pt nanoparticle agglomeration. Differences in Pt nanoparticle orientation, alignment, and the overall $\mathrm{Pt}$ agglomerate morphology on the highly graphitized carbons serves to accelerate coalescence in these systems. Efforts are ongoing in examining this effect in greater detail, as well as in the 3D characterization of the conditioned state of the different catalyst/support systems [8]. 
References:

[1] C Chen et al, Science 343 (2014), p. 1339.

[2] C Cui et al, Nano Lett. 12 (2012), p. 5885.

[3] S. I. Choi et al, Nano Lett. 13 (2013), p. 3420.

[4] X Huang et al, Science 348 (2015), p. 1230.

[5] B Y Xia et al, Chem. Eur. J. 16 (2010), p. 8268.

[6] K Schlögl et al, J. Electrochem. Soc. 159 (2012), p. B677.

[7] J C Meier et al, Beilstein J. of Nanotech. 5 (2014), p. 44.

[8] Research supported by Fuel Cell Technologies Office, Office of Energy Efficiency and Renewable Energy, U.S. DOE. Microscopy performed at ORNL's Center for Nanophase Materials Sciences, which is a U.S. DOE, Office of Science User Facility; instrumentation provided by the U.S. DOE Office of Nuclear Energy, Fuel Cycle R\&D Program, and the Nuclear Science User Facilities.

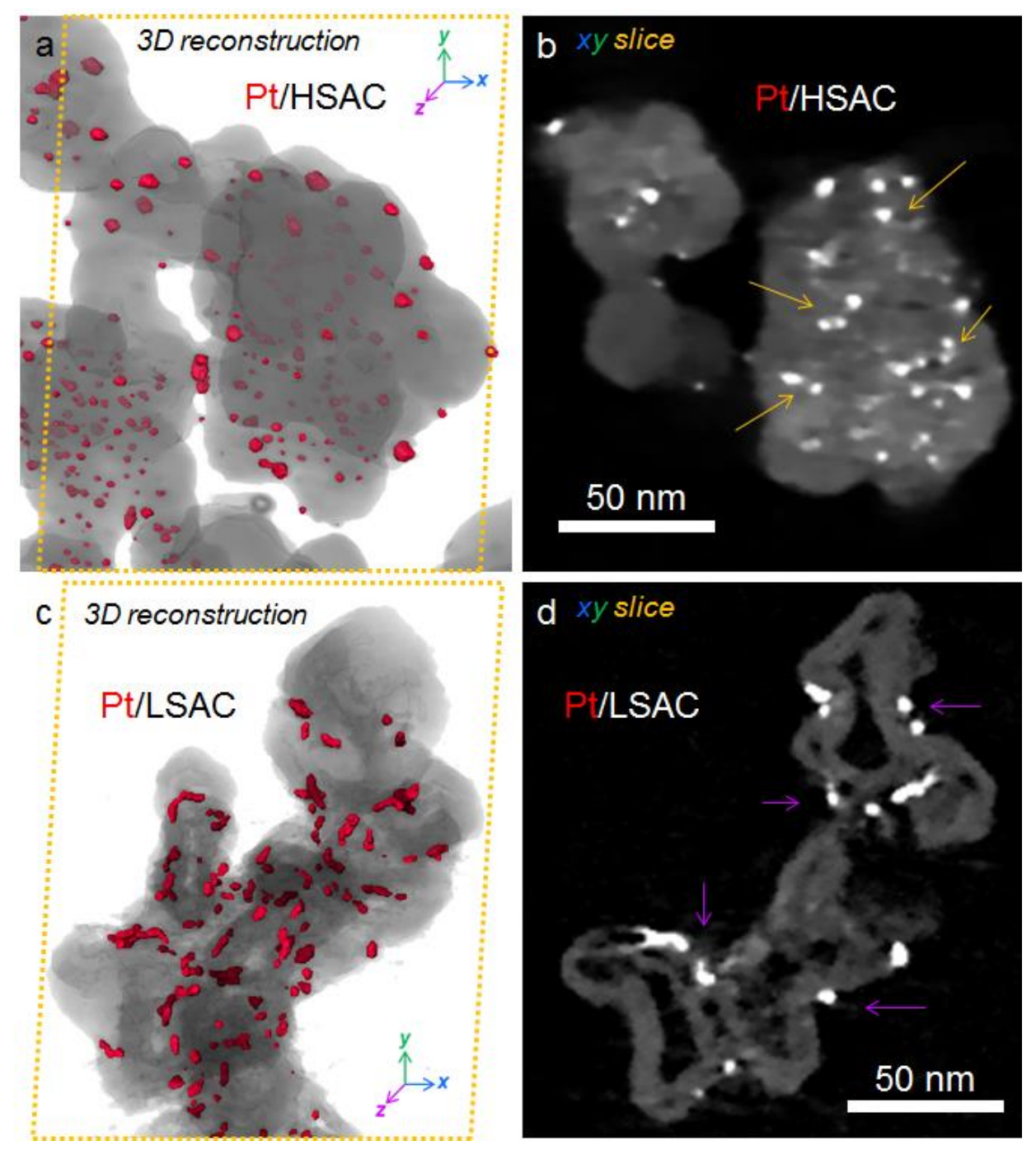

Figure 1. Electron tomography 3D rendering and cross-sectional slices of Pt on (a,b) HSAC and (c,d) LSAC graphitized carbon. Gold arrows show Pt particles occupying internal pore structure of HSAC (b), while purple arrows indicate Pt depositing at edge or defect sites near junctions of LSAC particles (d). 